\title{
Vivipary as a tool to analyze late embryogenic events in maize
}

\author{
D Durantini ${ }^{1}$, A Giulini ${ }^{1}$, A Malgioglio ${ }^{1}$, R Pilu ${ }^{1}$, R Tuberosa ${ }^{2}$, C Sanguineti ${ }^{2}$ and G Gavazzi ${ }^{1}$ \\ ${ }^{1}$ Dipartimento di Produzione Vegetale, Università degli Studi di Milano, Milano, Italia and ${ }^{2}$ Dipartimento di Scienze e Tecnologie \\ Agroambientali, Università di Bologna, Bologna, Italia
}

\begin{abstract}
In maize vivipary, the precocious germination of the seed while still attached to the ear, is the diagnostic phenotype of mutants, which are impaired in the biosynthesis or response to abscisic acid (ABA). Of the 15 genes so far described, 12 control specific steps in ABA biosynthesis, two mediate hormone response and one still has an undefined role. We have analyzed a collection of 25 independent $v p$ isolates with the aim of determining the degree of mutational saturation that has so far been reached. Of the 25 viviparous mutants complementation tested, 22 correspond to known loci: six are allelic to $v p 1$, another six to $v p 5$, one to $v p 7$, two to $v p 9$, six to $v p 10$ and one to $w 3$. The remaining three represent genes not previously identified. All mutants so far tested except rea
\end{abstract}

Keywords: maize; vivipary; $A B A$

\section{Introduction}

Germination is a crucial step of plant development that requires the accomplishment of specific events during the late phase of embryogenesis. These events are the acquisition of dehydration tolerance and the maturation process characterized by the accumulation of storage products. In many cultivated species, including maize, the mature dry seed is in a state of quiescence, characterized by a very low metabolic activity and an arrest of development imposed by unfavourable environmental conditions. Germination occurs when the suitable environmental conditions are established. In addition to quiescence, many seeds show a period of dormancy where the embryo does not germinate under otherwise favourable conditions. Seed dormancy is generally an undesirable trait in agricultural crops where rapid germination and growth are required. Presumably the trait 'seed dormancy', originally present in the wild ancestors of the cultivated plants, was lost during the process of plant domestication. Understanding the requirements for germination is an important prerequisite for plant improvement. A central role in the modulation of the events leading to embryo quiescence and dormancy is played by the phytohormone abscisic acid (ABA) (Finkelstein et al., 2002).

Correspondence: Professor G Gavazzi, Dipartimento di Produzione Vegetale, Università degli Studi di Milano, Via Celoria 2, 20133 Milano, Italia.

E-mail: giuseppe.gavazzi@unimi.it

Received 8 November 2007; revised 18 March 2008; accepted 18 March 2008 show a decrease in ABA content. As to the only two mutants (vp1 and rea) whose endogenous ABA content is not impaired, the reduction in sensitivity of the double mutant compared to the single ones suggests that the two genes control separate pathways in the ABA signal transduction. Some of the mutants in this collection have a characteristic incomplete germination that allows the embryo of the mature dry seed to resume germination. By exploiting this feature it is possible to infer, through a germination test, whether the mutant has been impaired in the acquisition of desiccation tolerance. This information provides the starting point for the dissection of the genetic basis of desiccation tolerance. Heredity (2008) 101, 465-470; doi:10.1038/hdy.2008.29
Abscisic acid exerts an important role in several physiological processes related to plant productivity (Zeevaart and Creelman, 1988).

In vegetative tissues, the increased ABA concentration induced by water deficit limits water loss through transpiration by inducing stomatal closure (Busk et al., 1999). In the seed, $A B A$ is thought to regulate several processes during late embryogenesis including the induction of seed dormancy, the accumulation of nutritive reserves and the acquisition of desiccation tolerance. These events are associated with the expression of specific sets of mRNA (Leung and Giraudat, 1998).

In maize vivipary, the precocious germination of the seed while still attached to the ear is the diagnostic phenotype of mutations impaired in the biosynthesis or the sensitivity of the embryo to abscisic acid. Because of their easily detectable phenotype, these mutants have been known for a long time. Eight non-allelic mutants were reported by Mangelsdorf (1926) and were later characterized genetically (Robertson, 1955).

The list of viviparous mutants has grown to include 15 genes: $v p 1, v p 2, v p 5, v p 7, v p 8, v p 9, v p 10, v p 12, v p 14, v p 15$, al1, y9, w3, rea and dek33 (McCarty, 1995). They can be grouped into three classes. Mutants $v p 1$ and possibly rea, referred to as response mutants (class 1), define genes not impaired in ABA biosynthesis. Class 2 includes mutants associated with reduced or suppressed carotenoid accumulation in both endosperm and vegetative tissues $(v p 2, v p 5, v p 7, v p 9, v p 12, a l 1, y 9$ and $w 3)$ as a result of a mutational block in early biosynthetic steps before the branching point that separates ABA and carotenoid biosynthesis (Singh et al., 2003). Class 3 includes vp8 and 
op14 which affect later steps of the biosynthesis (Schwartz et al., 1997) as well as vp10 and vp15 that regulate the synthesis of the molybdenum cofactor required for the last step in ABA biosynthesis (Porch et al., 2006; Suzuki et al., 2006). The relationship between dek33 and ABA has not yet been investigated. We have collected a number of viviparous mutants with the intent of ascertaining, by means of a complementation test, whether we can identify new genes involved in ABA metabolism or response or if mutational saturation has been reached. We have also attempted to establish whether some of these mutants are impaired for the capacity of the embryo to acquire desiccation tolerance.

\section{Materials and methods}

\section{Origin of the mutants}

All mutants of reference genes were obtained from the Maize Genetics Stock Center except vp1, a gift from Dr D Robertson. The origin of the mutants analyzed here is given in Table 1. They were originally isolated in different genetic backgrounds and were outcrossed once to $\mathrm{B} 73$ and W64A inbred lines to obtain vigorous $F_{2}$ ears as a source of material for the embryo rescue experiments. The rea mutant is included in this list because, even though previously described (Sturaro et al., 1996), its complementation pattern to known op mutants was not yet ascertained.

\section{Complementation test}

To test the allelic relationship between the 25 vp mutants in the collection and the op mutants reported in the literature, and here referred to as reference $v p$, we grouped the collection and reference mutants into two classes, that is, one with green seedling (class a) and another one (class b) with white seedling. We then made crosses of all mutants of the collection within each class, with all reference mutants of the same class. To perform this test, for each mutant in the collection, pollen of 5-10 + /op plants, whose heterozygous condition was ascertained by selfing, was applied to the silks of plants representing the selfed progeny of $+/ v p$ parents heterozygous for each of the reference mutants. More specifically, mutants in the collection belonging to class a) were tested against $v p 1, v p 8, v p 10, v p 14, v p 15$, rea and dek33 whereas each mutant of class b) was tested against $v p 2$, vp5, vp7, vp9, vp12, al1 and $y 9$. The resulting ears were then scored for visual evidence of segregation for vivipary.

\section{Isolation of rea vp double mutant}

To obtain homozygous rea op double mutants, we crossed rea/ rea females with op1/op1 male parents. The $\mathrm{F}_{1}$ progeny plants (Vp1/op1 Rea/rea) were selfed. Non viviparous homozygous rea seeds exhibiting red embryonic axis (rea/rea $V p 1 /$ - in genotype) were selected, planted and selfed. $\mathrm{F}_{3}$ ears segregating for one-quarter

Table 1 Description of the viviparous mutants under test and results of their complementation pattern to known $v p$ mutants

\begin{tabular}{|c|c|c|c|c|c|}
\hline $\begin{array}{l}\text { Provisional } \\
\text { symbol }\end{array}$ & Source & Origin & Phenotype & $\begin{array}{l}\text { Positive allelism } \\
\text { with }\end{array}$ & $\begin{array}{l}\text { New } \\
\text { designation }\end{array}$ \\
\hline \multicolumn{6}{|c|}{ (a) Viviparous mutants with green seedling } \\
\hline$v p^{*}-A$ & D Styles & Spm stock & Mutable aleurone in colored background & $v p 1$ & $v p 1-A$ \\
\hline$v p^{*}-B$ & D Styles & Spm stock & Mutable aleurone in colored background & op1 & vp1-B \\
\hline$v p^{*}-C$ & D Styles & Spm stock & Mutable aleurone in colored background & vp1 & vp1-C \\
\hline$v p^{*}-B 14$ & B Burr & EMS to seeds & Mutable aleurone in colored background & op1 & vp1-B14 \\
\hline$v p^{*}-426$ & B Burr & EMS to seeds & Normal & op1 & vp1-426 \\
\hline$v p^{*}-107$ & G Gavazzi & $d 5$ stock & Inger $^{\mathrm{a}}$ & op1 & vp1-107 \\
\hline$v p^{*}-374$ & B Burr & EMS to seeds & Enrolled leaves, Inger, callus ${ }^{b}$ & op10 & vp10-374 \\
\hline$v p^{*}-390$ & B Burr & EMS to seeds & Coloured aleurone, Inger, $\mathrm{des}^{\mathrm{c}}$ & op10 & vp10-390 \\
\hline$v p^{*}-D$ & D Styles & Spm stock & Seedling with adherent leaves & op10 & vp10-D \\
\hline$v p^{*}-105$ & R Pilu & EMS to seeds & Green tip of $\mathrm{Ea}^{\mathrm{d}}$, des, lethal seedling & op10 & vp10-105 \\
\hline$v p^{*}-108$ & G Gavazzi & Ac stock & Green tip of Ea, narrow leaves & op10 & vp10-108 \\
\hline$v p^{*}-109$ & R Pilu & spontaneous & Inger, frequently Sht $\mathrm{e}^{\mathrm{e}}$ seedling & op10 & vp10-109 \\
\hline$v p^{*}-404$ & B Burr & EMS to seeds & Enroled leaves, Inger & none & \\
\hline rea & G Gavazzi & EMS to seed & Red $\mathrm{Ea}$ & none & rea \\
\hline \multicolumn{6}{|c|}{ (b) Viviparous mutants with pale-green/white seedling } \\
\hline$v p^{*}-v i v 1$ & G Gavazzi & X-rays to pollen & Plumule unable to break pericarp & vp5 & vp5-viv1 \\
\hline$v p^{*}-v i v 2$ & G Gavazzi & $d 1$ stock & Normal & op5 & vp5-viv2 \\
\hline$v p^{*}-A \cdot V$ & A Viotti & Ac stock & Normal & op5 & vp5-AV \\
\hline$v p^{*}-102$ & M Racchi & Somaclonal variat. & Normal & op5 & vp5-102 \\
\hline$v p^{*}-104$ & R Pilu & EMS to seeds & Inger & op5 & vp5-104 \\
\hline$v p^{*}-110$ & G Gavazzi & a1 stock & Inger, des & op5 & vp5-110 \\
\hline$v p^{*}-100$ & G Gavazzi & $d 1$ stock & Inger & vp7 & vp $7-100$ \\
\hline$v p^{*}-106$ & R Pilu & EMS to seeds & Inger, des and frequently Sht seedling & op9 & vp9-106 \\
\hline$v p^{*}-430$ & B Burr & EMS to seeds & Normal & op9 & vp9-430 \\
\hline$v p^{*}-103$ & G Gavazzi & $d 2$ stock & Inger, distorted leaves & $w 3$ & $w 3-103$ \\
\hline$v p^{*}-366$ & B Burr & EMS to seeds & Inger, reduced leaf margin & none & \\
\hline
\end{tabular}

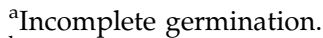

${ }^{\mathrm{b}}$ Tendency of immature embryos to yield callus.

${ }^{\mathrm{c}}$ Defective seedling.

${ }^{\mathrm{d}}$ Embryonic axis.

eShootless.
} 
viviparous seeds, rea/rea Vp1/vp1 in genotype, were the source of the double mutant.

\section{Embryo rescue of single vp1, rea and vp1 rea double mutants}

The sensitivity of single ( $v p 1$ and $r e a$ ) versus double (op rea) mutants to exogenous ABA was tested by excising immature (25 DAP: days after pollination) normal and mutant embryos obtained by selfing + /vp plants, and immediately transferring them to plant cell culture vessels (Phytotray Sigma, St Louis, MO, USA), containing MS medium (MS salts, $2 \%$ sucrose, $0.8 \%$. agar) in the absence or presence of ABA $50 \mu \mathrm{M}$ (mixed isomer, Merck, Whitehouse Station, NJ, USA).

Seedling elongation (from the first node to the tip of the last leaf) was determined after 8 days of culture in a growth chamber at $25^{\circ} \mathrm{C}$ with $14 / 10 \mathrm{~h}$ light/dark photoperiod.

\section{Desiccation tolerance of viviparous mutants}

This test was conducted either on dry seeds by selecting and germinating those mutants arrested in an early phase of germination (incomplete germination), as well as their normal sibs, or by comparing the germination rate of immature $v p$ embryos, before or after artificially induced desiccation. Immature $\mathrm{F}_{2}$ ears were left in $\mathrm{Na}$ hypochlorite: $\mathrm{dH}_{2} \mathrm{O}(1: 1 \mathrm{v} / \mathrm{v})$ solution for $30 \mathrm{~min}$ and then rinsed in sterile water. Mutant embryos on a segregating ear are recognizable because of incipient vivipary or pale yellow seed (for albino mutants). The $v p$ and normal sibling embryos were excised and transferred to plant cell culture vessels (Phytotray Sigma) on basal MS medium containing $2 \%$ sucrose solidified with $0.8 \%$ agar, or subjected to desiccation and a storage period of 60 days at $5{ }^{\circ} \mathrm{C}$ before transfer to the same medium.

Embryos were maintained in a growth chamber at $25^{\circ} \mathrm{C}$ with a $14 / 10 \mathrm{~h}$ light/dark photoperiod. Seedling elongation (from the first node to the tip of the third leaf) was determined after 8 days of culture.

For the drying treatment, embryos were laid between two disks of blotting paper within a sterile Petri dish and incubated in an oven at $35^{\circ} \mathrm{C}$ for $48 \mathrm{~h}$ with the lid open. At the end of treatment the dishes were sealed with Parafilm and conserved at $5{ }^{\circ} \mathrm{C}$ with silica gel at the bottom of the Petri dish, under the blotting paper.

Samples were conserved for 2 months until the start of the germination test on MS medium. For this purpose, embryos were maintained in a growth chamber at $25^{\circ}$ with $14 / 10 \mathrm{~h}$ light/dark photoperiod. After 10 days of culture, germination and seedling elongation were determined.

The parameters employed to define germination were protrusion of the primary root and/or the shoot.

Plant material for measurement of $A B A$ concentration Immature (25-30 DAP) mutant and normal embryos obtained by selfing $+/ v p$ plants, were surface sterilized and either stored at $-30^{\circ} \mathrm{C}$ or transferred to Phytotray Sigma vessels, in growth chambers at $24^{\circ} \mathrm{C}$ with a $16 / 8 \mathrm{~h}$ (light and dark) photoperiod. At the three-leaf stage, the third distal portion of the leaf lamina was collected from the second leaf of six plants per line. Leaf samples were immediately placed in plastic bags and stored at $-30^{\circ} \mathrm{C}$ until they were used.
The concentration of physiologically active, unconjugated ABA [2-cis(+)-ABA] was determined by a radioimmunoassay using an ABA-specific monoclonal antibody (MAC252; see Quarrie et al., 1988 and Tuberosa et al., 1998; available from Babraham Bioscience Technologies, Cambridge, UK) on samples of crude aqueous extracts of leaf disks (approximately $150 \mathrm{mg}$ fresh weight; dilution in distilled water $1: 10 \mathrm{w} / \mathrm{v}$ ) and following the procedures described in Tuberosa et al. (1994). Determination of ABA concentration was carried out with duplicate assays. The difference between duplicate assays of the same biological sample was usually within $10 \%$ of the average value. Values of $\mathrm{L}-\mathrm{ABA}$ are reported as ng $\mathrm{ABA} \mathrm{g}^{-1}$ fresh weight (f.w.).

\section{Results}

\section{Origin of the collection of viviparous mutants}

The viviparous mutants under test, 25 in total, have different origins, thus representing independent mutational events (Table 1). The majority of them have been recovered following chemical mutagenesis to seeds (11 mutants). Six were isolated in populations carrying an active transposon, but no attempt has been made to establish whether they are transposon tagged. Six are spontaneous in origin. One arose as a somaclonal variant, and one following pollen treatment with X-rays. They include mutants associated with reduced or suppressed carotenogenesis in both endosperm and seedling tissues (class 2) or without impaired carotenoid accumulation thus yielding yellow endosperm and green seedlings (classes 1 and 3 ). The complementation test with all available op mutants (Table 1) disclosed allelism of six mutants with $v p 1$, six with $v p 5$, one with $v p 7$, two with $v p 9$, six with $v p 10$ and one with $w 3$. Of the three mutants not showing allelism to any of the known $v p$ mutants, $v p^{*} 366$ may control as yet unidentified early steps of ABA biosynthesis, $v p^{*} 404$ could represent either a responsive or a biosynthetic mutant affecting a late step in ABA biosynthesis or synthesis of molybdenium cofactor, whereas rea with a normal endogenous ABA level can be considered a response mutant (Sturaro et al., 1996).

\section{Mutant segregation values}

The majority of the mutants behave in their transmission as single gene recessive mutants. However, for five of those allelic to $v p 10$ there is a significant $(P=0.01)$ deficit of mutants over the expected one-quarter, presumably as a result of gametophytic selection. Two mutants, vp5-102 and $v p 10-108$, are recovered with a very low frequency (5-10\%) in the selfed progeny of heterozygous parents. These results can be due to low penetrance, or to the simultaneous segregation of modifiers of vivipary expressivity or to the existence of duplicate factors. In fact, the $v p$ mutants under test are in a heterogeneous genetic background. As an example, we noticed that the characteristic green shoot tip developed by vp10-105 in its native background is lost when the mutation is introduced into different genetic backgrounds.

Endogenous ABA content in embryo and seedling tissues The ABA level of immature embryos is reported in Table 2. The data refer to a selected sample (13) of the 25 
Table 2 Endogenous ABA content (ng/g f.wt) of immature (25-30 DAP) embryos and of leaf tissues from sib mutant and wild-type embryos cultured on MS medium for eight days

\begin{tabular}{|c|c|c|c|c|}
\hline \multirow[t]{2}{*}{ Mutant } & \multicolumn{2}{|c|}{ Embryo } & \multicolumn{2}{|c|}{ Seedling } \\
\hline & $+^{\mathrm{a}}$ & $m$ & + & $m$ \\
\hline rea & $116.0^{\mathrm{b}}$ & 119.0 & 320.0 & 280 \\
\hline$v p 1$ & 120.0 & 80.0 & 28.0 & 22 \\
\hline vp1-426 & 122.1 & 21.5 & 23.1 & 21 \\
\hline vp1-B14 & 426.3 & 32.6 & 27.1 & 24. \\
\hline$v p 1-B$ & 231.4 & 44.5 & - & - \\
\hline vp5-102 & 112.8 & 13.9 & 13.2 & 7.8 \\
\hline$v p 7-100$ & 232.8 & 24.3 & 17.8 & 4.0 \\
\hline vp10-D & 281.9 & 18.4 & 22.5 & 10.9 \\
\hline op10-374 & 60.0 & 11.8 & 17.1 & 16.0 \\
\hline vp10-390 & 47.3 & 12.1 & 12.2 & \\
\hline vp10-105 & 102.2 & 25.5 & 23.2 & 12.3 \\
\hline$v p^{*}-366$ & 85.7 & 13.5 & 32.8 & 10 \\
\hline$v p^{*}-404$ & 117.9 & 10.6 & 24.9 & 15 \\
\hline
\end{tabular}

${ }^{a_{+}}$and $m$ stand for wild-type and viviparous siblings.

bexpressed as ng/g dry wt.

mutants of the collection where vivipary could be unequivocally detected on immature seeds. ABA was reduced when compared to wild-type sibs in all mutants tested with the exception of rea and $v p 1$ (the latter here reported as a control) where it remained the same. As compared to wild-type, reduction in mutant ABA content was also observed in the seedling tissues of the mutants with values ranging from a slight reduction to about one-half or one-third of the wild-type values.

\section{ABA sensitivity of single vp1, rea and vp1 rea double mutants}

Contrary to the other mutants, rea is not impaired in its endogenous ABA content, a characteristic shared by vp1. Accordingly we compared the ABA sensitivity of the double mutant $v p 1$ rea to that of single mutants. To assay ABA sensitivity of the mutants tested we analyzed the response of the embryos to exogenous ABA. The response of each mutant and normal sibling was expressed as reduction in elongation of eight-day-old seedlings grown on MS medium supplemented with ABA $\left(50 \mu\right.$ mole $\left.^{-1}\right)$ over siblings grown on the same medium devoid of ABA. On the basis of this test the double mutant appeared significantly less sensitive than either mutant taken alone (Figure 1), a result that suggests a cumulative effect of $v p 1$ and rea on the degree of ABA sensitivity. This would be expected if the two genes act on separate pathways of the signal transduction pathway. This conclusion corroborates previous results (Sturaro et al., 1996), indicating that vp1 and rea differ in their pattern of ABA-inducible genes.

\section{Acquisition of desiccation tolerance in vp mutants}

Incomplete germination is a trait observed only in some mutants in our collection. It consists of a subtle elongation of the embryonic axis that fails to break through the pericarp. This trait is somehow variable in its expression and is occasionally associated with accumulation of anthocyanins (Figure 2). Since incomplete germination suggests an early arrest in the precocious germination process associated with vivipary,

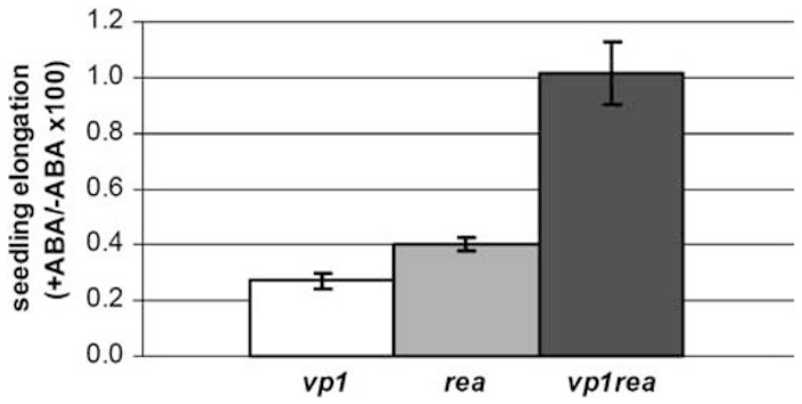

Figure 1 Effect of exogenous ABA $50 \mu \mathrm{M}$ on growth of single (vp1 and rea) versus double (vp rea) mutants. Growth is expressed as seedling elongation on media with $\mathrm{ABA} /$ seedling elongation on media without $A B A \times 100$. Length measurements were taken after 10 days of culture of immature embryos (30 DAP). Error bars indicate \pm s.e.

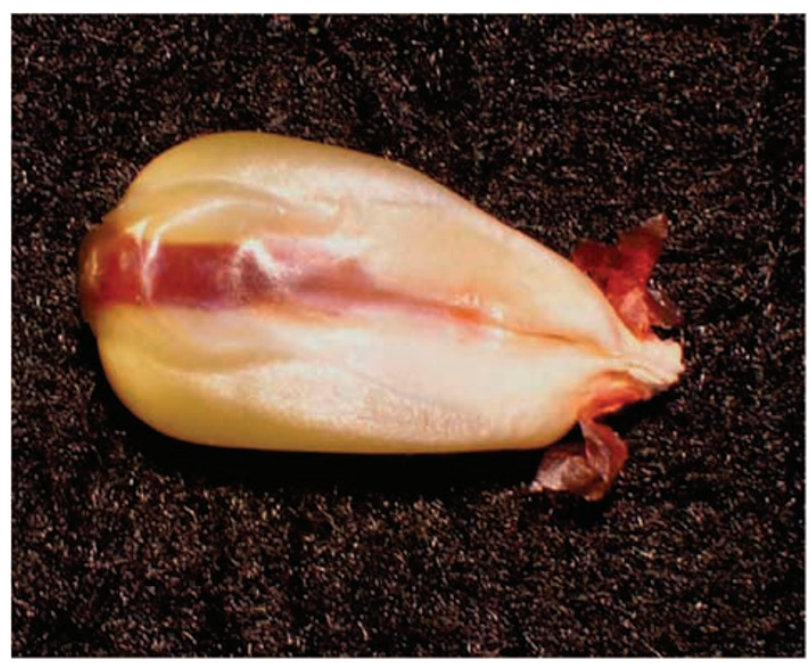

Figure 2 Mature seed with incomplete germination. Note the elongation of the embryonic axis and the accumulation of anthocyanins.

we tried to establish if these mutants have maintained desiccation tolerance and could accordingly be rescued. For each of the mutants under test, as well as for known $v p$ mutants, the capacity to maintain desiccation tolerance was tested by germinating a sample of seeds with incomplete germination. On the basis of the germination results obtained by analysing the mutants (17) that exhibit incomplete germination, we could make the following distinctions: mutants exhibiting partial maintenance of desiccation tolerance were vp10, vp10-109, $v p 10-374, v p 10-\mathrm{D}, w 3-103$ and $v p^{*} 366$. These mutants germinated with variable frequency, yielding abnormal seedlings with distorted growth and leaf shape; alternatively they protruded the primary root but lacked a shoot. On the other hand, vp1, vp1-107, vp2, vp5-104, $v p^{*} 404$ and $w 3$ are desiccation intolerant whereas $v p 12$, vp14, vp9-106 vp10-105 and rea, are completely tolerant with about a $100 \%$ germination and a normal seedling morphogenesis (Table 3). To confirm that incomplete germination might be a valid criterion to infer acquisition of desiccation tolerance, immature (25-30 DAP) mutant embryos showing this trait were either subjected 
Table 3 Germination as mature dry seed and seedling morphogenesis of viviparous mutants

\begin{tabular}{lrll}
\hline $\begin{array}{l}\text { Mutant } \\
\text { symbol }\end{array}$ & $\begin{array}{r}\text { Germination } \\
(\%)^{\mathrm{a}}\end{array}$ & $\begin{array}{l}\text { Seedling } \\
\text { morphogenesis }\end{array}$ & $\begin{array}{l}\text { Dessication } \\
\text { tolerance }^{\mathrm{b}}\end{array}$ \\
\hline$v p 1$ & 0.0 & $\mathrm{ND}^{\mathrm{c}}$ & None \\
$v p 1-107$ & 0.0 & ND & None \\
$v p 2$ & 0.0 & ND & None \\
$v p 5-104$ & 0.0 & ND & None \\
$v p 9-106$ & 90.0 & Impaired & Good \\
$w 3$ & 1.2 & Normal & Very poor \\
$w 3-103$ & 37.3 & Impaired & Medium \\
$v p 10$ & 64.0 & Impaired & Medium \\
$v p 10-109$ & 16.7 & Impaired & Poor \\
$v p 10-D$ & 10.0 & Impaired & Poor \\
$v p 10-105$ & 100.0 & Impaired & Good \\
$v p 10-374$ & 51.4 & Impaired & Medium \\
$v p 12$ & 97.1 & Normal & Good \\
$v p 14$ & 95.7 & Normal & Good \\
$r e a$ & 100.0 & Normal & Good \\
$v p^{*}-366$ & 10.0 & Impaired & Poor \\
$v p^{*}-404$ & 4.3 & Impaired & Very poor \\
\hline
\end{tabular}

${ }^{a}$ Confidence limits below \pm 10 percent of the value $(95 \%$ confidence interval).

${ }^{\mathrm{b}}$ Germination \% >80\%: good; 40-60\%: medium; $10-20 \%$ : poor; $<5 \%$ : very poor.

'ND: no seedling developed.

For each mutant $n>100$.

to premature desiccation treatment before culturing or directly cultured on fresh medium. The data obtained indicate (Table 4) that premature desiccation does not allow recovery of germination of vp366*,vp404* and w3-103 mutants but only a partial recovery of vp10-374. This finding is in agreement with results obtained by germinating mature embryos exhibiting incomplete germination.

\section{Response of mutants to osmotic stress in vegetative tissues}

To investigate whether the different response of the mutant embryos in terms of desiccation tolerance is correlated with their response to stress conditions in vegetative tissues, we grew mutant $v p 366^{*}$ and wild-type sibs on media supplemented with mannitol, an osmoticum mimicking water-stress (Figure 3). Under this treatment both mutant and wild-type seedlings showed a severe reduction of shoot elongation and, to a less extent, of primary root elongation. Therefore, the mutant is not impaired in its sensitivity to the osmoticum. Similar results were obtained with mutants $v p-103^{*}$, vp-404* and $v p 10-374$ (data not shown).

\section{Discussion}

The main reason for analyzing this collection of mutants was to determine whether mutational saturation for genes involved in ABA biosynthesis has been reached. This was achieved by establishing, through a complementation test, the allelic distribution of the $v p$ mutants among known $v p$ and then using the unassigned mutants to test the feasibility of identifying new genes controlling specific steps in ABA biosynthesis or response. The results obtained indicate that at least three mutants cannot be assigned to known op genes and thus appear
Table 4 Effect of drying immature (25-30 DAP) embryos on their germination capacity

\begin{tabular}{|c|c|c|c|c|c|}
\hline \multirow[t]{3}{*}{ Segregation for } & \multirow[t]{3}{*}{$D A P$} & \multicolumn{4}{|c|}{ Germination (\%) } \\
\hline & & \multicolumn{2}{|c|}{ Fresh } & \multicolumn{2}{|c|}{ Dried $^{\mathrm{b}}$} \\
\hline & & $+^{c}$ & $m$ & + & $m$ \\
\hline$v p^{*}-366$ & 25 & 72.2 & 100 & 100 & 0 \\
\hline$v p^{*}-404$ & 25 & 100 & 100 & 75 & 0 \\
\hline w3-103 & 29 & 100 & 100 & 95 & 0 \\
\hline vp10-374 & 24 & 97.1 & 93.3 & 100 & 78 \\
\hline
\end{tabular}

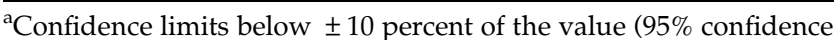
interval).

${ }^{\mathrm{b}} 37^{\circ} \mathrm{C}$ for $48 \mathrm{~h}$.

${ }^{c}+$ and $m$ refer to wild-type and viviparous siblings.

Homozygous mutant and normal embryos obtained on a selfed $+/ v p$ plant were excised and either transferred on MS medium or dried and cultured on the same medium after 60 days of storage at $5{ }^{\circ} \mathrm{C}$ For each determination at least 50 embryos were cultured.

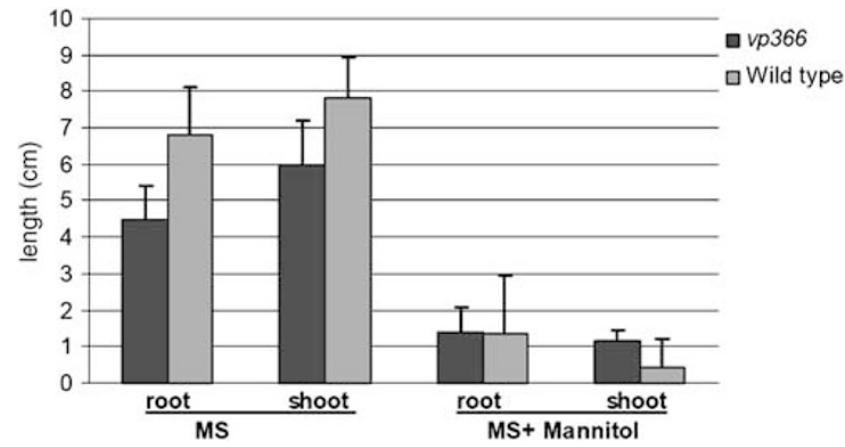

Figure 3 Effect of mannitol upon root and shoot elongation of $v p * 366$ mutants and their wild-type siblings. Immature seeds were grown on MS medium with or without mannitol $(0.3 \mathrm{M})$. Root and shoot length of mutant and wild type siblings were determined after 7 days of culture. Error bars indicate \pm s.e.

to define new genes involved in ABA synthesis or signal transduction.

A similar survey conducted by McCarty (1996) on viviparous mutants with green seedlings in a population of selfed ears in the presence of an active transposon (Mu-DR) led to the identification of three alleles of $v p 1$, seven of $v p 10$, two duplicate factors and five unassigned. Altogether, these results suggest that we have not yet reached the saturation point for genes involved in the ABA pathway. In this context it will be interesting to find out which step or steps is controlled by the unassigned mutants.

The segregation of the mutants here analyzed deserves some comments. For some mutants, an attempt to establish their segregation ratio was made difficult by the finding that these values were significantly different from ear to ear. This observation suggests either the presence of duplicate factors, modifiers or the effect of changes in activity of a transposon inserted into the gene under study. A significant deficit of the mutants over the expected 1:3 ratio was the rule for the alleles at the vp10 locus, a result that suggests a function of its gene product at the gametophytic level. Mutant deficit in this case would be the result of gametophytic selection, a hypothesis that we will test.

Characterization of the mutant collection in terms of ABA content indicates that all the mutants tested show a 
decrease, to a different extent, in its level compared to their wild-type sibs. However, the mutants show a wide range of variability in their endogenous ABA content. The significant reduction of endogenous ABA observed in all tested $v p 1$ alleles ( $v p 1-426, v p 1-\mathrm{B} 14$ and $v p 1-\mathrm{B})$ is surprising if we consider that $v p 1$ is not involved in ABA biosynthesis. If we consider that all mutant embryos are germinating at the stage of embryo collection, we can assume that the mutant embryos are catabolizing ABA at a developmental stage when normal embryos are accumulating ABA. If so, embryonic ABA levels are not comparable since the developmental stage of mutant and normal embryos are different. However, when they are comparable, as in the case of rea, the ABA level of normal and mutant embryos are the same. It is remarkable that among the known viviparous mutants with reduced ABA sensitivity, only $v p 1$ and rea are unaltered in their ABA content. Our results, showing a reduction in sensitivity of the double mutant when compared to the single ones, seem to suggest that the two genes are involved in the control of separate pathways of the ABA signal transduction.

Desiccation tolerance of the embryo, an important feature related to both fitness as well as productivity of the plant, is acquired by the embryo between 20 and 25 days after pollination (Bochicchio et al., 1988). The incomplete germination shared by some of the tested mutants suggests that the mutation can be associated to loss of desiccation tolerance in some mutants whereas in others such tolerance is completely or partially maintained. These results have been confirmed by testing the germination rescue of immature mutant embryos subjected to premature desiccation. This observation is of some relevance since it provides the starting material for dissecting the genetic basis of desiccation tolerance, a particularly important trait in view of the increasing occurrence of drought episodes that can heavily curtail maize survival in the field during the early stages of growth. In addition, we found that all mutants exhibiting partial desiccation tolerance are impaired, to a different extent, in their morphogenesis whereas those with normal tolerance yield normal seedlings. These results seem to suggest a link between desiccation tolerance and morphogenesis that should be further investigated.

\section{Acknowledgements}

We thank Dr Burr for critically reading the article and the editor as well as three anonymous reviewers for their constructive criticism. This work was supported by the Ministero dell'Istruzione dell'Università e della Ricerca (COFIN- 2001 to GG).

\section{References}

Bochicchio A, Vazzana C, Raschi A, Bartels D, Salamini F (1988). Effect of desiccation on isolated embryos of maize. Onset of dessication tolerance during development. Agronomie 8: 29-36.

Busk PK, Borrell A, Kizis D, Pagès M (1999). Abscisic acid perception and transduction. In: Hooykass PIJ, Hall MA, Libbenga KR (eds). Biochemistry and Molecular Biology of Plant Hormones. Elsevier Sci: Amsterdam, The Netherlands, pp 491-512.

Finkelstein RR, Gampala SSL, Rock CD (2002). Abscisic acid signaling in seeds and seedlings. Plant Cell 14: S15-S45.

Leung BV, Giraudat JJ (1998). Abscisic acid signal transduction. Ann Rev Plant Physiol Plant Mol Biol 49: 199-222.

Mangelsdorf PC (1926). The genetic and morphology of some endosperm characters in maize. Conn Agr Exp Bull 279: 513-614.

McCarty D (1995). Genetic control and integration of maturation and germination pathways in seed development. Ann Rev Plant Physiol Plant Mol Biol 46: 71-93.

McCarty DR (1996). Genetic analysis of seed maturation and germination pathways in maize. In: Wang T, Cuming A (eds). Embryogenesis: The Generation of a Plant. BIOS Scient Publ: Oxford, UK, pp 133-141.

Porch TG, Tseung CW, Schmeltz EA, Settles AM (2006). The maize viviparous10/viviparous13 locus encodes the $C n x 1$ gene required for molybdenum cofactor biosynthesis. Plant J 45: 250-263.

Quarrie SA, Whitford PN, Appleford NEJ, Wang TL, Cook SK, Henson IE (1988). A monoclonal antibody to (S)-abscisic acid: its characterization and use in a radioimmunoassay for measuring abscisic acid in crude extracts of cereal and lupin leaves. Planta 173: 330-339.

Robertson DS (1955). The genetics of vivipary in maize. Genetics 40: 745-765.

Schwartz SH, Tan BC, Gage DA, Zeevaart JAD, McCarty DR (1997). Specific oxidative cleavage of carotenoids by vp14of maize. Science 276: 1872-1874.

Singh M, Lewis PE, Hardeman K, Baj L, Rose JKC, Mazourek M et al. (2003). Activator mutagenesis of the pink scutellum1/ viviparous7 locus of maize. Plant Cell 15: 874-884.

Sturaro M, Vernieri P, Castiglioni P, Binelli G, Gavazzi G (1996). The rea (red embryonic axis) phenotype describes a new mutation affecting the response of maize embryos to abscisic acid and osmotic stress. J Exp Bot 47: 755-762.

Suzuki M, Settles AM, Tseung CW, Li QB, Latshaw S, Wu S et al. (2006). The maize viviparous15 locus encodes the molybdopterin synthase small subunit. Plant J 45: 264-274.

Tuberosa R, Sanguineti MC, Landi P (1994). Abscisic acid concentration in the leaf and xylem sap, leaf water potential, and stomatal conductance in drought-stressed maize. Crop Sci 34: 1557-1563.

Tuberosa R, Sanguineti MC, Landi P, Salvi S, Conti S (1998). RFLP mapping of quantitative trait loci controlling abscisic acid concentration in leaves of drought-stressed maize (Zea mays L.). Theor Appl Genet 97: 744-755.

Zeevaart JAD, Creelman RA (1988). Metabolism and physiology of abscisic acid. Ann Rev Plant Physiol Plant Mol Biol 39: 439-473. 